\title{
Commentary
}

\section{Comment on article "Does pain confound interpretation of neuropsychological test results?"}

\author{
Paul Green* \\ Neurobehavioural Associates, Edmonton, AB, Canada
}

Nicholson, Martelli and Zasler conclude their review by stating "Future studies should be informed by an understanding of the psychology of chronic pain, as well as more rigorous neuropsychological methodology". We can only agree with this conclusion because there has been a lack of rigour in past research, much of which has simply assumed that low test scores in patients are equivalent to cognitive impairment. It is possible that chronic pain does affect cognition but we cannot be sure of this until the effects of incomplete effort on tests have been controlled properly.

In this review, Nicholson, Martelli and Zasler did not quote the study in which people with chronic complaints after whiplash injuries were given an effort test, the Amsterdam Short Term Memory Test (ASTM, Schmand, 1997). Based on their ASTM results, 61\% of patients were found to be exaggerating their memory impairment on formal testing. Hence, their other ability test results would be presumed to be invalid. On the other hand, the latter study was quoted by Hart et al. [5], in a review of neuropsychological functioning in chronic pain patients. These authors concluded that tests of response bias and motivation should be used in evaluating chronic pain patients, who are involved in litigation or seeking wage replacement benefits. Logically, effort tests should be used not only in clinical cases but also in all group studies of chronic pain patients. If not, the results of any study of cognition and

*Address for correspondence: Paul Green, Ph.D., Neurobehavioural Associates, 201 17107-107 Avenue, Edmonton, Alberta, Canada T5S 1G3. Tel.: +1 780484 5550; E-mail: paulgreen@shaw. ca. chronic pain must be considered to be of very doubtful validity. This applies to all the studies of chronic pain quoted in the review by Nicholson, Martelli and Zasler.

The evidence summarised by Larrabee [7], in relation to mild head injury, suggests that persistent complaints are ten times more likely to be a result of symptom exaggeration than actual cognitive impairment resulting from head injury. Allen et al. [1] indicated that symptom exaggeration, shown by failing well validated effort tests, was higher in their patients with chronic pain than in cases of mild head injury. Gervais et al. [2] found a $42 \%$ failure rate on the Computerised Assessment of Response Bias or CARB [1] and the Word Memory Test [3] in a group of chronic pain patients. However, in a subsequent group, who were warned in advance that CARB was unaffected by pain and emotional distress and that failing the test would imply poor effort, the failure rate on CARB dropped to only $6 \%$. Failure on the WMT, about which they were not warned, did not change from the baseline. These data suggest a very major influence of incomplete effort on cognitive tests in chronic pain patients. If uncontrolled, a $40 \%$ incidence of exaggeration could easily give the false impression that cognitive impairment is present in a group of patients with chronic pain.

Using 904 patients of various diagnoses, Rohling et al. [8] showed that $53 \%$ of the variance in scores on 43 neuropsychological tests was explained by scores on effort tests. In contrast, years of education and age had a very minor impact on test scores, explaining only $11 \%$ and $4 \%$ of the variance, respectively. In this study, the effects of incomplete effort in suppressing test scores were far greater than the effects of severe brain injuries. In the vast majority of studies of chronic pain, effort has not been controlled, but it has been found to be significant in the few studies which have taken it into account. Therefore, the most economical 
hypothesis, at present, would be that most or all of the apparent impairment of cognitive abilities in chronic pain patients is an artefact of failing to remove invalid test results, produced by cognitive exaggeration.

\section{References}

[1] L.M. Allen, R.L. Conder, P. Green and D.R. Cox, CARB '97 Manual for the Computerized Assessment of Response Bias, CogniSyst, Inc., Durham, NC, 1997.

[2] R. Gervais, P. Green, L.M. Allen and G. Iverson, The effects of coaching on Symptom Validity Testing in chronic pain patients presenting for disability assessments, Forensic Neuropsychology (in press)

[3] P. Green, L.M. Allen and K. Astner, The Word Memory Test:
A User's Guide to the Oral and Computer-Administered Forms, US Version 1.1, CogniSyst, Inc., Durham, NC, 1996.

[4] P. Green, G.L. Iverson and L. Allen, Detecting malingering in head injury litigation with the Word Memory Test, Brain Injury 13(10) (1999), 813-819.

[5] R.P. Hart, M.F. Martelli AND N.D. Zasler, Chronic pain and neuropsychological functioning, Neuropsychol Rev 10(3) (2000), 131-149.

[6] G.L. Iverson, P. Green and R. Gervais, Using the Word Memory Test to detect biased responding in head injury litigation, Journal of Cognitive Rehabilitation 17 (1999), 4-8.

[7] G.J. Larrabee, Neuropsychology in personal injury litigation, Journal of Clinical and Experimental Neuropsychology 22 (2000), 702-707.

[8] M.L. Rohling, P. Green, L.M. Allen and P.R. Lees-Haley, Effect Sizes of Impairment Associated with Symptom Exaggeration versus Definite Traumatic Brain Injury, Archives of Clinical Neuropsychology 15 (2000), 839. 